\title{
Touching the past: Haptic Augmented Reality for Museum Artefacts
}

\author{
Mariza Dima ${ }^{1}$, Linda Hurcombe ${ }^{2}$, Mark Wright ${ }^{3}$ \\ ${ }^{1}$ University of Edinburgh, Edinburgh, UK \\ mdima@exseed.ed.ac.uk \\ ${ }^{2}$ University of Exeter, Exeter, UK \\ L.M.Hurcombe@exeter.ac.uk \\ ${ }^{3}$ Liverpool John Moore University, Liverpool, UK \\ M.W.Wright@1jmu.ac.uk
}

\begin{abstract}
In this paper we propose a novel interaction technique that creates the illusion of tactile exploration of museum artefacts which are otherwise impossible to touch. The technique meets the contextual necessity, often requested by museum curators, to background technology and to direct the focus of the museum visitor's experience to the artefact itself. Our approach relies on the combination of haptic interaction and the adaptation of a well-known illusion that enables museum visitors to make sense of the actual physical nontouchable artefact in an embodied way, using their sensory and motor skills. We call this technique Haptic Augmented Reality.
\end{abstract}

Keywords: Museum, haptics, touch, authenticity, haptic augmented reality

\section{Introduction}

Touch is part of a larger complex of senses which interrelates mental and bodily processes, the haptic sense. Haptic exploration is a fundamental experience that assists people in perceiving and making sense of the physical world around them. The sensory information of the museum exhibits, particularly surface texture and material, is particularly important for museum visitors since the artefacts themselves are the center of the social, educative and entertaining experience of a museum visit. Whilst the value of touch experiences can be debated there is a growing literature on sensory engagement in museums which seeks to redress the imbalance which has traditionally allowed the visual sense to dominate [10] [14].

The emphasis on touch experiences in heritage settings and museums has emerged as a distinctive trend from this exploration [6] [24] alongside discussions of sensory perceptions of materiality as social constructs within both past societies and our own with the two not necessarily coinciding [15]. The value of touch has thus received nuanced debate within museums studies and has been explored as a related set of sensory concepts [23]. A feature of the role of touch has been the emotional connections of objects and people and the charisma of objects where it is possible to see ancient artefacts displayed in museums as having an extended object biography bringing them into our contemporary cultural context [10] [16].

Within digital technologies and computer applications a number of views and directions have emerged but the heritage sector in general is seeing a range of developments in the applications of haptic and virtual presentations of objects within museums [11] [5] [8]. In the networking cluster described more fully below the concerns of heritage sector curators, exhibitions officers and conservators was not so much on the value of adding touch experiences to the museum experience but on how to balance curating the objects whilst providing touch experiences. The charisma of objects and the desire of people to touch them were acknowledged. Well-known objects were seen as particularly problematic. That is why the focus of the installations discussed here was one of the Lewis chess pieces as these objects are amongst the most popular artefacts in the whole of the collections within the National Museums Scotland. 


\section{Design process}

Virtual handling of museum artefacts lies within a complex context of different professional practices, technological development and end-user needs. As part of the Science and Heritage programme funded by EPSRCAHRC, Linda Hurcombe led an international project bringing researchers from different disciplines into a networking cluster focused on 'Touching the Untouchable: increasing access to archaeological artefacts by virtual handling'.

It was therefore appropriate to adopt a design led user-centred approach that would bring the many experts involved in a creative dialogue. The interaction technique we present in this paper was one of the outcomes of two design-led workshops that took place for two days each over the period of six months. Many of the keyissues that are related to curatorial practice and technological development were described and discussed in the first workshop, and prototype ideas were developed and presented in a second workshop six months later. From the first meeting of this group it was evident that there were multiple issues faced by the heritage sector and many potential ideas for solutions.

The workshops involved 26 participants from 19 institutions and 6 countries. Disciplines included archaeology, conservation and curation together with art and interaction design, computer science and haptic humancomputer interfaces. Representatives from small and national museum collections, artifact specialists, the National Trust, Historic Palaces, the Royal National Institute for the Blind attended and all presented different points of emphasis offering a richly textured insight into professional practices. The transdisciplinary nature of the first workshop allowed key issues to be raised and discussed from a plethora of perspectives, while design sessions involved participants in collaborative hands-on work and cultivated a number of ideas that were developed as first prototypes and evaluated in the second workshop.

On the first day participants gave short position presentations on their work and the key issues as they saw them. There were also demonstrations of museum specimens and haptic technology. The second day consisted of a plenary session where stakeholders discussed a broad range of themes and opportunities arising from the previous day. Topics included haptic device capabilities, archaeology research agendas, curation and end users and the potential benefits of virtual handling.

Key issues that were raised included:

- Haptic installations may deflect interest away from the ancient items on show both physically and conceptually.

- The technology for virtual touch needs not to overwhelm its physical setting e.g. a museum gallery and must be able to cope with the visitor numbers (i.e. size of machine, noise, ease of use)

- Can haptic experiences get away from the computer desktop?

- Products and solutions could be expected to be diverse according to the kind of user and their setting. Rapidprototyping could be explored for its practical issues and scope.

- Financial mechanisms for public display varied and virtual technologies need to be assessed against the robustness of device and costs to set up and expertise to maintain them.

The focus of the present paper is on two of many more prototypes which were developed in response to these key issues and which were presented in the second workshop for testing and evaluation. They were well received by the stakeholders and after some corrections were made, they were deployed and evaluated in two museums: the National Museum of Scotland in Edinburgh and the Orkney Museum in Kirkwall. These evaluations and prototypes flowed from the first networking grant which pursued them to proof of concept stage. More recent work was undertaken as part of a second grant also led by Hurcombe within the Science and Heritage programme which allowed them along with some of the other ideas to be given more extensive public trials and development. The full range of installations developed is covered elsewhere [17] but here the focus is on one famous object presented in two contrasting ways.

\section{The prototypes}

The museum exhibit that was used is an iconic 12th century Scottish artefact known as the Lewis Queen chess. The artefact is displayed in the National Museum of Scotland behind a glass case. Both prototypes use the same visual illusion but employ different media, one digital and one non-digital. The visual illusion is borrowed from the theatre tradition and is called Pepper's Ghost. A large sheet of glass is placed between the audience and 
the stage. A ghostly image of an actor below the stage is then projected onto the glass giving the illusion that a ghost is on stage with the actors. Using the Pepper's Ghost illusion we employed two different media, a 3-D printed replica of the chess piece and a haptic device. Both used the glass of the museum case itself as a reflective surface thus ensuring that the focus was the real object or the haptic experience.

The replica was created by laser scanning the original artefact, mirroring it to the original (i.e. lateral inversion of the scan data before printing) so that the user's experience would match the object in the case. The need to present mirror images as part of virtual reality and co-location interfaces is part of virtual reality issues [3] [18]. The replica was then painted black so that it would absorb light thereby reducing its reflection in the glass case. The replica is placed facing the chess piece at an equal distance from the display glass (Fig.1). When a user places her hands on the replica and concentrates her gaze at the original piece behind the glass, she can see her hands reflected in the glass apparently touching the real artefact in the display case (Fig.2). Because she sees the actual artefact (and her hands) and touches the replica she experiences the sensation that she is actually touching the artefact itself. The illusion is further strengthened by placing a cover over the replica to shield it from the user's direct gaze. This cover also contains a light to illuminate the user's hands so that their reflection is brighter.

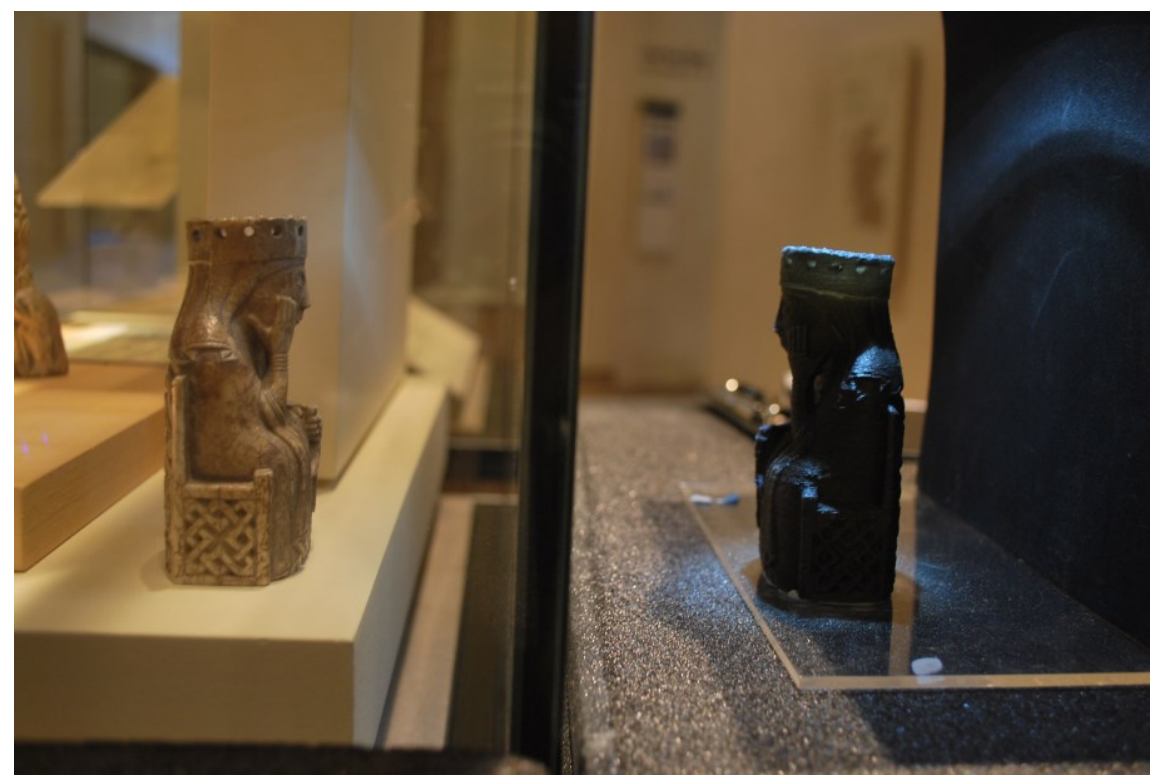

Fig. 1. The Lewis Chess piece behind the glass and the mirrored 3-D printed replica

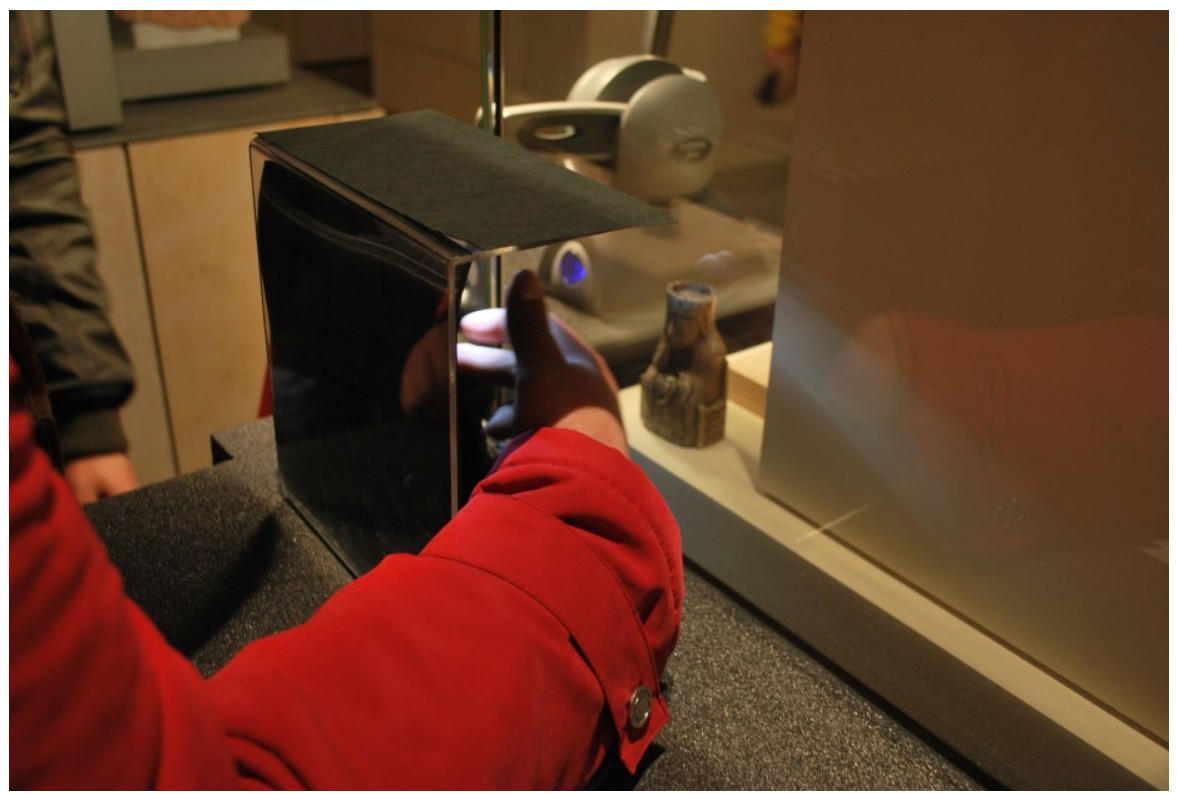

Fig. 2. Visitor interaction with the replica. Her gaze is concentrated at the original artefact behind the glass 
The second prototype uses the same illusion but employs a Sensable ${ }^{\mathrm{TM}}$ Omni 6DoF haptic device instead of the user's hands. The haptic device is placed outside the display case and positioned towards the left of where the replica was so that the reflection of the pen-like stylus of the haptic device is positioned close to the artefact in the display glass. Instead of a replica, a haptic model created from the laser scan of the artefact is algorithmically positioned into the haptic device's workspace at an equal distance from the display case (Fig.3). The haptic version is invisible but the model can be traced and felt in the physical space by moving the stylus using the same combined visual and haptic feedback as with the replica prototype.

This is a novel way of using a haptic device for immersing museum visitors into a deep understanding of the museum exhibits. In [19] museum visitors explore the surface of a digital daguerreotype case from the collection of the Natural History Museum of Los Angeles County. Similarly, the Haptic Museum, developed in the University of Southern California, is a haptic interface that allows museum visitors to examine virtual museum artefacts with a haptic device [21]. The 'Museum of Pure Form' is a virtual reality system that allows the user to interact with virtual models of 3-D art forms and sculptures using haptic devices and small-scale haptic exoskeletons $[1,2]$. The Senses in Touch II, which was installed in the Hunterian Museum in Glasgow, was designed to allow blind and partially-sighted museum visitors, particularly children, to feel virtual objects in the collection via a PC and Wingman haptic mouse [13]. The projects described above have used detailed virtual models of the museum artefacts and allowed the visitor to explore them with the haptic technology. Our goal was to diverge from the computer screen, and use the haptic technology in a way that evokes direct haptic interaction with the physical artefact without actually touching it providing the illusion of doing so.

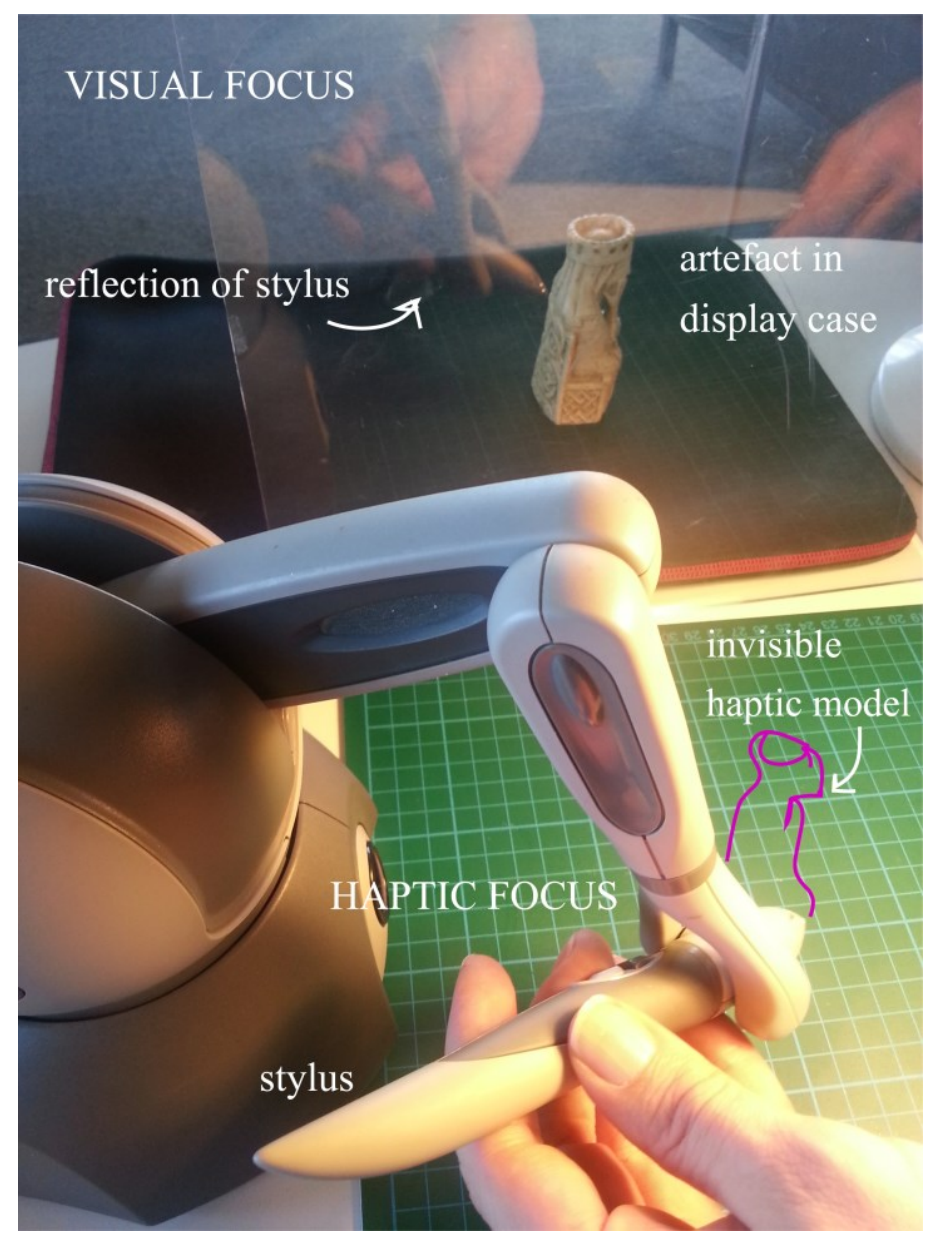

Fig. 3. The haptic device prototype

Equally, the Pepper's ghost technique has been used in the Virtual Showcase, a mirror-based interface for viewing real artefacts augmented with virtual geometry [3]. In Virtual Showcase no touch is used as the focus is on adding additional virtual objects and other elements onto the real object. ARToolkit-based optical tracking is used for tracking the user's head movement in real time to ensure collocation with the virtual components. Head 
tracking was important in the Virtual Showcase because of the virtual geometry. In our prototype no tracking of the head is required. As long as the replica or the invisible haptic model are placed in exactly the same orientation and same distance from the surface of the glass case, the illusion of co-location is preserved under all translations and rotations of the viewing angle which preserve a direct line of sight from the viewpoint through the case wall to the real artefact.

\subsection{Embodiment and sense-making}

Our interaction with the world around us is embodied and multi-modal and we make sense of the world by enacting in it. Enactive knowledge is direct, in the sense that it is natural and intuitive, based on the perceptual array of motor acts. The goal of both prototypes was to create an embodied and immersive experience for the visitors in order to provide a sense of authenticity for the ancient artifact. Embodiment and situated cognition places interaction at the center of meaning making and extends the concept of mind to include the body and environment [25], [7], [22].

The illusion of manipulation that the presented interaction technique creates can be explained as directly analogous to a classic experiment in situated cognition and perceptual plasticity [20]. A subject places their hand on a table hidden behind a partition. On the other side of the partition they can see a dummy hand. Their real hand and dummy hand are touched by fingers of a researcher which are synchronized to touch in the same place at the same time but the subject can only see the finger that touches the dummy hand not the one that touches their real hand. After a short time the subject perceives the dummy hand to be their own. In the two prototypes, the visual stimulus of the researchers visible finger is replaced by the reflection of the users own hands or haptic probe. The synchronized haptic stimulus is provided by the subjects' fingers touching the replica or felt through the haptic device when it collides with the invisible virtual $3 \mathrm{~d}$ model of the artefact. The haptic device enables haptic exploration and sense-making through multi-modal motor action which makes them an enactive interface [12]. The combination of haptic and visual feedback in both prototypes enriches the array of senses during the interactive experience and creates more dimensions of embodiment than having only visual cues.

\section{Evaluation}

The replica based prototype is created using digital technology of laser scanning, 3D modelling and rapid prototyping but is itself a non-digital tangible interface. It offers a simple, robust and apparently technology free interaction backgrounding technology entirely. The haptic device is a digital interface and uses the same laser scan to build its virtual haptic model. Our intention is to compare these two prototypes in a real museum setting.

It is a challenge to evaluate user experience that is closely related to embodied, tacit understandings, such as in this case. The evaluation goals concern subjective opinions of visitor focus, degree of engagement and phenomenological experience. As these goals are subjective and not easily mapped to any objective quantifiable factor our evaluation was a combination of gathering feedback, verbal or written, and close observation of the way the visitors used the interface, their gestural motions as well as the social interaction among them during their experience.

We were present at all time next to the exhibits and were interacting with the visitors, observing their interaction with the artefact through both interfaces, having informal discussions about their experience, often using probing questions, and triggering their reflection when something would break their experience. The visitors were then asked if they would like to fill in a qualitative questionnaire. They were then asked to rate with a 5 point Likert Scale (from strongly disagree, disagree, neutral, agree or strongly agree) the following statements:

1. It is important to engage more senses than the visual as part of the museum experience.

2. The installation/replica gave a sense of how the ancient object would feel.

3. The installation/replica was straightforward to use.

4. Because of the installation/replica there was a better understanding of the ancient objects.

5. Overall, the installation/replica enhanced the museum experience. 
They were also prompted to write any more detailed comments.

Both prototypes attracted a number of visitors most of whom were eager to discuss with the researchers and learn more about the project as well as its technical details. A large number of visitors would stay for a considerable time and explore the possibilities of the interface, giving us verbal feedback and discussing details of its use. As only one person at a time could use each prototype, visitors would gather around and watch, conversing with one another and with the researchers until it was their turn. Children were particularly drawn to the installations and they were the ones to stay longer and explore it. This shows that both interfaces can enable playful engagement.

There were 60 questionnaire responses over the two days installation at the National Museum of Scotland and the two days at the Orkney Archaeological Museum. The initial empirical results demonstrated the potential of both prototypes to provide a novel embodied experience of untouchable artefacts. Visitors' comments were very positive for both prototypes but particularly for the replica.

The rapid prototype installation successfully produced the sense of haptic exploration of the chess piece in a natural and simple way. The setup synchronised the visitors' visual and haptic cues, and consequently, their interaction with the replica was directly translated as interaction with the real statue. One visitor commented 'As I felt it, I felt like I was touching the one in the reflection and not the replica'. Another one said that it 'feels real and that you feel more connected to its history'.

One drawback of the replica installation was the double image of the hands on the glass created by the refraction of the light on the perplex glass. A few visitors found this a bit distracting, though not detrimental to the whole experience. The double image can be corrected in future versions by calculating optical parameters based on a specific position where the visitor will be standing. Another interesting comment made by three visitors was that the texture of the replica should be improved to match as much as possible the material of the original piece. This would improve the perception of the exhibited piece and will be taken forward in future designs.

The main drawback of the haptic interface that was reported from the discussions and written comments was that the haptic device could not provide a detailed outline of the statue. Most visitors could not easily perceive the fine details of the statue with the stylus. One reason for this was the size and detail of the exhibit. The installation could work very well for larger objects or small objects with little details. The lack of precision can be slightly improved by developing a more sensitive collision detection system between the haptic device controller and the haptic geometry which allows for more detailed tracing of the carved details. One of the future tests is to use an artefact with few details and compare user responses, both verbal and bodily, with those received in this study. The aim will be to investigate the extent to which the interface conveys sufficient realism starting from relatively simple objects. The lack of detailed information was also attributed to the single-point contact of the device compared to the multi finger touch of the hands.

\section{Discussion}

The visitors agreed unanimously that the combination of visual and haptic cues gave a much better sense of the object, and increased the sense of authenticity in comparison to just viewing it in its case. Because of the size of the chess piece, some visitors commented that a seated position would bring the piece on their eye height and reduce the fatigue from standing up and using the interface.

The comparison between the haptic device and the replica showed that the multi-finger tactile interaction with the replica produced considerably richer information than the single-point contact of the haptic device. The surface texture and material of an artefact plays a significant role when exploring haptically an object and the Phantom haptic device cannot provide this level of haptic rendering at sufficient quality. The authors acknowledge that a single contact point is relatively little information compared to the amount of haptic sensations that a finger can give by touching a texture or a complex surface. The key issue is whether small additions to the museum experience are worthwhile.

The two different installations allow comparative assessments on such issues which add to the current literature on heritage applications of haptic and virtual experiences with objects. Our research juxtaposed two different experiences to the same object allowing for direct comparisons. The relative costs, maintenance issues, and ease of use, as well as the visitor feedback and comments all pointed in favour of the computer mediated but physical replica compared to the active haptic device. Yet without the trial this was not a predictable outcome as the readiness of visitors to engage with the virtual reflection and the coalignment of visual hand image with touch experience was one of the key trial results. In contrast, the haptic pen could have been handled by visitors in much the same way as a simple wooden stick could be drawn across the face of a textured object to probe aspects of its morphology and textures. 
The trial results certainly relate to cognitive perception but they also relate back to the clear directive of the end-users: to hide the technology and for it not to overwhelm the visitors. Visitors were clearly more comfortable aligning real touch of a hidden replica co-located with a virtual reflection than working an obvious computer-related largely unfamiliar device. Though the design of the pen was fairly robust and easy to use as a device once shown, not many visitors knew about haptic pens and the device by its nature could not be hidden. These are important aspects in the willingness of visitors to engage with unfamiliar technologies versus their desire to interact with objects within glass cases. Such results have been highlighted in other research [5] [11] reinforcing our conclusion that the familiarity of the touch experience at the level of embodied practice can affect visitor perceptions but that as haptic devices are developed and become more mainstream experiences they can more easily be applied. Still, these statements are based on observing the visitors' readiness to engage with the installations and from some visitor comments about preferences between the two. It is more difficult to attribute this to familiarity versus immediacy which the replica presents stronger than the haptic pen whether produced by $3 \mathrm{D}$ printing or other means.

Finally, the 3D print technologies are reducing in price and are now not so expensive. Compared to using a PHANToM, the 3D printed replica is a lower cost solution.

\section{Further developments}

Further innovations have been explored within the project to give better textures and tactile qualities such as weight and more personal interactions but these raise many other issues which require fuller discussion elsewhere [17].

The results from the comparison between the two interfaces indicated that the haptic device prototype provides a less complex sensation of the artefact. However, there is ample scope for the use of haptic devices within this setup if the interaction mechanism is enriched with dynamic elements and other modalities, for example with sound feedback, extra touchable geometry, explanations, and texture all of which can be dynamic, personalized information. While this can be equally possible with the replica (e.g. by using depth cameras to calculate the hands position), the implementation through a haptic device is much easier and cost-effective.

Another development that is particularly for the haptic device prototype is to use the interface with museum artefacts that have missing parts as the device can be used to feel the invisible missing piece. In addition, a draw function can be implemented through which users can draw extra geometry. In [9] an early research on this process is presented, and the Virtual Showcase [3] that was mentioned in the literature review also allows the presentation of stereoscopic images overlaid on top of real objects. We envisage that this study will have numerous applications in museum research as well as learning.

\section{Conclusion}

We have presented a novel haptic interaction paradigm which gives the impression of direct haptic interaction with museum artefacts in their display cases. The prototypes solve the problem of technology taking focus from the artefact as attention is not on a graphic display or replica but the real artefact itself. The approach was tested in a real Museum environment and was found to provide enhanced engagement with a real precious artefact. Compared to the digital prototype, the non-digital conveyed richer sensory information about the artefact during interaction. However, the digital interface offers the opportunity for easily adding extra interactive elements that can enhance immersion. While much remains to be done, our work shows that the technique we developed has the potential of becoming a useful way of evoking multimodal embodied exploration of intangible artefacts, with significant educative and economic advantages for museums and similar exhibition and learning spaces.

\section{Acknowledgements}

The research reported here was part of the Science and Heritage programme and used funding from EPSRCAHRC as part of for a network cluster grant Touching the Untouchable and an AHRC development grant on Touching the Past both led by Linda Hurcombe; Mark Wright was CI on the latter. The research could not have happened without the substantial commitment and support shown by Dr Alison Sheridan (Principal Curator of Early Prehistory) of National Museums Scotland who came to the first workshop, hosted the second and who 
was involved in all the trials reported here. She facilitated access to the Lewis chess pieces case, arranged permissions and dealt with numerous practical and conceptual issues. The Orkney Museum staff also hosted the trial and we particularly thank Sheila Wilson, and Janette Park as curators as well as project researchers Lynda Aiano and Penny Cunningham. Dr Ian Summers (Physics, Exeter) particularly developed the idea of using pepper's ghost alongside the authors listed here and used them in another project installation. He, together with his former research student Dr Matt Philpott contributed to the success of the public trials of all of the installations.

\section{References}

1. Bergamasco, M., Frisoli, A., and Barbagli, F.: Haptics technologies and cultural heritage applications. In Proceedings of Computer Animation pp. 25-32 (2002)

2. Bergamasco, M., Avizzano, C., Di Pietro, G., Barbagli, F., and Frisoli, A.: The museum of pure form: system architecture. In Proceedings of 10th IEEE International Workshop on Robot and Human Interactive Communication, pp.112-117. (2001)

3. Bimber, O., Fröhlich, B., Schmalstieg, D., and Encarnação, L.M.: The Virtual Showcase, IEEE Computer Graphics \& Applications, vol. 21, no. 6, pp. 48-55 (2001)

4. Botvinick, Cohen J.: Rubber hands 'feel' touch that the eyes see, Nature 391, 756, (1998)

5. Brewster, S. A.: The impact of haptic 'touching' technology on cultural applications. Digital applications for cultural heritage institutions pp. 273-284 (2005)

6. Chatterjee C., Helen .J. (eds): Touch in Museums: policy and practice in object handling, Oxford: Berg (2008)

7. Clark, A.: Being There: putting brain, body and world together again, MIT Press (1998)

8. Contreras, F., Farjas M., and Melero F.J. (eds): Fusion of Cultures, In Proceedings of the $38^{\text {th }}$ Annual conference on Computer Applications and Quantitative methods in Archaeology, Granada, 2010, Oxford: BAR International Series 2494. (2010)

9. Dima, M., Arvind, D. K., Lee, J., and Wright, M.: Haptically extended augmented prototyping. In Proceedings of 7th IEEE/ACM International Symposium on Mixed and Augmented Reality pp. 169-170. (2008)

10. Dudley, S. (ed): Museum Materialities: Objects, Engagements and Materialities, London Routledge (2009)

11. Figueroa, P., Coral, M., Boulanger, P., Borda, J., Londono, E., Vega, F., Prieto, F., Restrepo D.: Multi-modal exploration of small artifacts: an exhibition at the Gold Museum in Bogota. In Proceedings of the 16th ACM Symposium on Virtual Reality Software and Technology. ACM (2009)

12. Froese, T., McGann, M., Bigge, W., Spiers, A., and Seth, A. K.: The enactive torch: A new tool for the science of perception. IEEE Transactions on Haptics 5, 4, pp.365-375 (2012)

13. Gibson, E., Penfold-Ward, J., Tasker, S., Williamson, J., and Wood, C.: Senses in touch II, University of Glasgow, Thirdyear project report (2001)

14. Gosden, C. Phillips, R., Edwards, E. (eds): Sensible Objects: Colonialism, Museums and Material Culture, Oxford; Berg (2007)

15. Hurcombe, L.: A sense of materials and sensory perception in concepts of materiality, World Archaeology 39:532-545 (2007)

16. Hurcombe, L.: Archaeological Artefacts as material Culture, London; Routledge. (2008)

17. Hurcombe, L., Sheridan, A., Summers, I., Wright, M., Dima, M., Philpott M.: in preparation, Touching the Past.

18. Jang, J.S., Jung, G.S., Lee, T.H. Jung, S.K.: Two phase calibration for a mirror metaphor augmented reality system, In Proccedings IEEEE 102(2) (2014)

19. Lazzari, M., McLaughlin, M. L., Jaskowiak, J., Wong, W., Akbarian, M.: A Haptic Exhibition of Daguerreotype Cases for USCs Fisher Gallery. Prentice Hall (2002)

20. Matthew, B., and Cohen, J.: Rubber hands 'feel' touch that eyes see. Nature 391 (1998)

21. McLaughlin, M. L., Sukhatme, G., Hespanha, J., Shahabi, C., Ortega, A., \& Medioni, G.: The haptic museum. Proceedings of the EVA 2000 Conference on Electronic Imaging and the Visual Arts, Florence, Italy. (2000)

22. Noe, A. Action in perception. MIT Press (2006)

23. Patterson, M.: The Senses of Touch, Oxford: Berg. (2007)

24. Pye, E. (ed): The Power of Touch: Handling objects in museums and heritage contexts Left Coast Press (2008)

25. Varela, F.: The Embodied Mind. MIT Press (1992) 